\title{
Estudo do sonograma do ducto venoso em fetos com centralização hemodinâmica: avaliação de repercussões perinatais
}

\author{
Study of ductus venosus in fetuses with brain sparing reflex: evaluation of perinatal outcomes \\ Paulo Roberto Nassar de Carvalho ${ }^{1}$, Maria Elisabeth Lopes Moreira², Renato Augusto Moreira de Sá ${ }^{3}$, \\ Ynesmara Cosmos ${ }^{4}$, Laudelino Marques Lopes ${ }^{5}$
}

\section{RESUM0}

\begin{abstract}
Objetivo: avaliar a associação da relação sístole ventricular/atrial(S/A) do ducto venoso (DV) com resultados perinatais em fetos prematuros com centralização de fluxo à dopplervelocimetria. Métodos: o estudo foi delineado como um estudo observacional, transversal, com os dados colhidos de forma prospectiva. A relação S/A do DV foi estudada em 41 fetos centralizados com idade gestacional (IG) entre 25 e $33^{\mathrm{a}}$ sem ana completa, no período de novembro de 2002 a julho de 2005. Os recém-nascidos foram acompanhados até o $28^{\circ}$ dia pós-parto na UTI da Clínica Perinatal Laranjeiras, buscando-se complicações neonatais. A população de estudo foi dividida em dois grupos a partir do resultado do DV. Foram incluídos no grupo normal os fetos com relação S/A menor ou igual a 3,6 e no grupo alterado aqueles com valores de S/A maiores que 3,6. A comparação entre os grupos foi realizada com os testes estatísticos de Mann-Whitney, $\chi_{2}$ e exato de Fisher. Todos os resultados foram considerados estatisticamente significativos se $p<0,05$. Foram avaliados a IG ao nascimento, peso ao nascere índice de Apgarm enor que 7 no $5^{\circ}$ minuto. Os parâmetros perinatais avaliados foram: natimortalidade, neomortalidade, convulsão, hemorragia intraventricular, necessidade de surfactante, uso de respirador, disfunção miocárdica, enterocolite necrotizante e perm anência na UTI após o período neonatal. Resultados: dos 41 fetos incluídos, $26(63,4 \%)$ não apresentaram alteração da relação S/A doDV, ao passo que $15(36,6 \%)$ fetos cursaram com a relação S/A do DV alteradas $(>3,6)$. Não houve diferença significativa entre os grupos quanto à $\mathrm{IG}$ ao nascim ento e A pgar $<7$. Encontrou-se associação significante entre o grupo com DV alterado e mortalidade neonatal ( $\mathrm{p}=0,049$; teste exato de Fisher). Quanto às dem ais variáveis estudadas não foi observada diferença entre os dois grupos. Conclusão: os nossos resultados sugerem que a anorm alidade do fluxo no DV detectada pela dopplervelocimetria não está associada a resultados perinatais adversos, além da mortalidade neonatal. Esta associação pode ser considerada estatisticamente significante, porém com valor próximo ao limite $(\mathrm{p}=0,049)$. Quando excluídos os fetos com peso de nascim ento inferior a 400 gramas, não é mais observada a associação entre o DV e neomortalidade $(\mathrm{p}=0,37)$. A alteração da relação S/A do DV não apresentou, em nossa amostra, associação com mortalidade perinatal em fetos prematuros viáveis.
\end{abstract}

PALAVRAS-CHAVE: Fluxometria por laser-doppler; Sangue fetal; Sofrimento fetal

\section{ABSTRACT}

Purpose: to evaluate the relationship between S/A ratio in ductus venosus (DV) and perinatal outcomes in fetuses with brain sparing reflex. Methods: the study was designed as an observational, sectional study with prospectively collected data. Forty-one fetuses with brain sparing reflex and gestational age between 25 and 33 weeks were studied between November 2002 and July 2005. The newborns were observed during the neonatal period in the intensive care unit of "Clínica Perinatal Laranjeiras" in order to find adverse outcomes. The study population was divided into two groups according to DV assessment. In the normal group all the fetuses with S/A ratio values of 3.6 or less were included, and in the abnormal group the fetuses with values of S/A ratio greater than 3.6. The statistical analysis was performed by the Mann-Whitney Utest, $\chi^{2}$ test and Fisher exact test. The results were considered significant when $\mathrm{p}<0.05$. Gestational age, birth weight and Apgar score less than 7 at 5 min were evaluated. Perinatal outcome parameters were: intrauterine death, neonatal mortality, seizures, intraventricular hemorrhage, leukomalacia, need of surfactant, mechanical ventilation, myocardical failure, necrotizing

Centro Pré-Natal de Diagnóstico e Tratamento (CPDT) da Clínica Perinatal Laranjeiras - Rio de Janeiro (RJ), Brasil.

1 Médico do Centro Pré-Natal de Diagnóstico e Tratamento da Clínica Perinatal Laranjeiras - Rio de Janeiro (RJ), Brasil.

2 Médica neonatologista da Clínica Perinatal Laranjeiras - Rio de Janeiro (RJ), Brasil.

3 Médico do Centro Pré-Natal de Diagnóstico e Tratamento da Clínica Perinatal Laranjeiras - Rio de Janeiro (RJ), Brasil.

4 Médica do Centro Pré-Natal de Diagnóstico e Tratamento da Clínica Perinatal Laranjeiras - Rio de Janeiro (RJ), Brasil.

5 Diretor de Medicina Materno-Fetal da Clínica Perinatal Laranjeiras - Rio de Janeiro (RJ), Brasil.

Correspondência: Paulo Roberto Nassar de Carvalho

Rua das Laranjeiras 445 Subsolo - Laranjeiras - 22240-002 - Rio de Janeiro - RJ - Telefone/FAX: (21) 2556-0022 (r. 141 e 142) - e-mail: paulonassar@cpdt.com.br 
enterocolitis, and length of stay in the intensive care unit. Results: among the assessed 41 fetuses, 26 (63.4\%) showed normal DV S/A ratio and the other $15(36.6 \%)$ developed an abnormalDV S/A ratio (>3.6). There was no statistically signicant difference between the groups according to gestational age at delivery and Apgar $<7$. The only significant association was between abnorm al D V S/A ratio and neonatal death ( $\mathrm{p}=0.049$; Fisher's exact test). No statistically significant association was observed for the other studied variables. Conclusions: our results suggest that abnormal DV blood flow detected by Doppler examination is not associated with adverse perinatal outcomes, except for neonatal mortality. This association may be considered statistically borderline $(\mathrm{p}=0.049)$. Excluding fetuses with birth weight less than $400 \mathrm{~g}$, there was no other association between DV and neonatal mortality. The abnorm al DV S/A ratio w as not associated, in our study, with perinatal mortality in viable preterm fetuses.

KEYWORDS: Laser-doppler flowmetry; Fetal blood; Fetal distress

Introdução

A decisão do melhor momento para intervir em gestação pré-termo comprometida por centralização de fluxo sangüíneo à dopplervelocimetria permanece difícil e objeto de diversos debates entre especialistas. Até o momento, não existe nenhuma medida terapêutica capaz de reverter o quadro de insuficiência placentária e seu progressivo insulto sobre o feto. A interrupção da gestação surge como alternativa para aquele grupo de pacientes com prognóstico fetal mais reservado ${ }^{1}$. No entanto, a antecipação do momento do parto, de forma inapropriada, pode ser extremamente danosa em gestações prematuras, em especial aquelas com menos de 32 semanas de idade gestacional (IG) ${ }^{2}$.

Tentar prolongar ao máximo a gestação, até o ponto em que não se observem danos fetais, parece ser objetivo razoável para que o concepto se beneficie da minimização dos riscos da prematuridade sem que ocorra acometimento efetivo de seus órgãos e sistemas ${ }^{1}$.

A dopplerfluxometria da artéria umbilical (AU) tem se mostrado eficaz na identificação e no prognóstico perinatal de gestações de alto risco, segundo ensaios clínicos controlados ${ }^{3,4}$. As alterações de fluxo sangüíneo fetal, causadas pela hipoxemia secundária à insuficiência úteroplacentária, são identificadas à dopplervelocimetria como aumento da resistência vascular na artéria umbilical e decréscimo dessa resistência no sistema nervoso central. Esse mecanismo de defesa fetal visa à perfusão preferencial de sangue bem oxigenado, oriundo do ducto venoso (DV), para o cérebro e outros órgãos vitais como coração e supra-renais, e recebe o nome de centralização de fluxo sangüíneo quando a resistência da AU supera a da artéria cerebral média (ACM) ${ }^{5}$.

A proposta de estudo dos vasos venosos pela dopplerfluxometria veio da observação, já há uma década, que alterações do Doppler arterial em fetos gravemente acometidos por crescimento intrauterino restrito (CIUR) eram seguidas de modifi- cações do fluxo venoso precordial (DV e veia cava inferior) ${ }^{6}$.

A dopplerfluxometria do DV tem se mostrado importante ferramenta na predição de complicações perinatais relacionadas à asfixia, em grupos de fetos portadores de CIUR precoce ${ }^{7-10}$. A relação sístole ventricular/contração atrial (relação S/A) do DV é considerada como bom marcador para hipoxemia em fetos com CIUR, em análise de sangue fetal obtida diretamente por cordocentese ${ }^{11}$.

O presente estudo tem por objetivo avaliar a associação da relação S/A do DV com resultados perinatais em fetos prematuros com centralização de fluxo ao Doppler. A identificação das variáveis associadas ao DV pode fornecer informação adicional para programação do momento de interrupção destas gestações, questão até hoje não totalmente respondida.

\section{Métodos}

O estudo foi delineado como estudo observacional, transversal, com os dados colhidos de forma prospectiva. Foram examinadas 41 gestações de fetos com centralização de fluxo sangüineo entre novembro de 2002 e julho de 2005 na unidade de ultra-sonografia da Clínica Perinatal Laranjeiras (CPL). Os critérios de inclusão foram: idade gestacional entre 25 e 33 semanas completas de gravidez, confirmada por exame ultra-sonográfico realizado antes da $20^{\mathrm{a}}$ semana; índices de pulsatilidade da artéria umbilical acima do $95^{\circ}$ percentil para a IG ${ }^{12}$; fetos com centralização de fluxo sangüíneo à dopplervelocimetria (indice de pulsatilidade da AU maior que da ACM); gestação única com ausência de malformações (confirmada no período pós-natal); parto cesáreo na ausência de trabalho de parto ativo. Complicações referentes ao parto, que poderiam influenciar no resultado perinatal (ex: hemorragias, dificuldade de extração), foram consideradas como critério de exclusão. Não houve exclusão de ne- 
nhuma paciente da população de estudo. As 41 pacientes participantes correspondem ao total de gestantes e fetos examinados na CPL no período de estudo que preenchiam os critérios de inclusão do mesmo.

A dopplerfluxometria colorida foi realizada com o aparelho Volusom 730 (GE Medical Systems, USA), usando transdutor convexo de 2-5 MHz. O filtro de alta freqüência foi ajustado para sensibilidade de $100 \mathrm{~Hz}$. O sonograma da AU foi obtido por meio da insonação de três segmentos do cordão umbilical: próximo à inserção abdominal, alça livre e próximo à inserção placentária. Foi considerada a média aritmética destes três segmentos. A obtenção do sonograma da ACM foi realizada conforme técnica previamente descrita ${ }^{13}$.

A dopplervelocimetria do ducto venoso foi obtida com a gestante em posição de semi-Fowler, em período de repouso absoluto do feto. Sua insonação foi realizada na origem do vaso, a partir da identificação do sinus portal em corte de secção transversal ou médio-sagital do abdome feito por meio de ultra-sonografia em 2 dimensões e da dopplervelocimetria colorida ${ }^{14}$. A imagem foi registrada quando ao menos 5 ondas de velocidade de fluxo foram obtidas com padrão característico do DV e com sinal constante. Essa abordagem garante um coeficiente de variação menor que $10 \%{ }^{15-17}$. Foram mensuradas a velocidade de pico durante a sístole ventricular (S) e a velocidade correspondente à contração atrial (A) e calculada a relação $\mathrm{S} / \mathrm{A}$. Todos os fetos tiveram a última insonação do DV pelo menos 24 horas antes do parto, realizada pelo pesquisador principal. Considerou-se a dopplervelocimetria de DV anormal quando a relação S/A foi maior do que dois desvios-padrão da média para a nossa população (relação $\mathrm{S} / \mathrm{A}>3,6)^{18}$.

Duas categorias foram criadas a partir do resultado da insonação do DV. No grupo DVnor, foram incluídos os fetos sem alteração da relação S/A do DV (relação S/A menor ou igual a 3,6). O grupo DValt foi reservado àqueles com relação S/A do DV alteradas (relação $\mathrm{S} / \mathrm{A}>3,6$ ). Os dados referentes às variáveis estudadas foram colhidos na sala de parto e durante todo o período neonatal (28 dias de nascido). As variáveis estudadas ao nascimento foram IG, peso e índice de Apgar menor que 7 no $5^{\circ}$ minuto de vida. Os desfechos perinatais avaliados foram: natimortalidade, neomortalidade, convulsão, hemorragia intraventricular, necessidade de surfactante, uso de respirador, disfunção miocárdica, enterocolite necrotizante e permanência na UTI por período superior ao neonatal.

A interrupção da gestação foi influenciada por doença materna (pré-eclâmpsia grave, síndrome HELLP) ou deteriorização do bem-estar fetal, avaliada pela dopplervelocimetria arterial, cardiotocografia e perfil biofísico do feto. A decisão sobre o término da gestação coube exclusivamente ao médico pré-natalista, sem que houvesse interferência direta do pesquisador.

Para comparação entre os grupos, as variáveis demográficas contínuas dos RN foram analisadas com o teste U de Mann-Whitney. Os testes de $\chi^{2}$ e exato de Fisher foram aplicados para analisar as associações entre as variáveis categóricas. Todos os resultados foram considerados estatisticamente significativos se $\mathrm{p}<0,05$. O estudo estatístico foi realizado utilizando-se o programa SPSS Statistical Software, version 10.0 (SPSS, Chicago, IL, USA).

O protocolo de estudo foi aprovado pelo Comitê de Ética em Pesquisa do Instituto Fernandes Figueira/Fundação Oswaldo Cruz. Consentimento pós-informado por escrito foi obtido de todas as participantes.

\section{Resultados}

Das gestantes estudadas, $20(48,8 \%)$ apresentaram pré-eclâmpsia como doença de base, duas $(4,9 \%)$ sindrome HELLP e seis $(14,6 \%)$ eram portadoras de hipertensão crônica. No grupo DVnor foram incluídos 26 fetos $(63,4 \%)$ sem alteração do DV e no grupo DValt, 15 fetos $(36,6 \%)$ com alteração da relação S/A do DV. A Tabela 1 representa a IG e o peso ao nascimento, bem como índice de Apgar $<7$ no $5^{\circ}$ minuto.

Tabela 1 - Idade gestacional, peso e índice de Apgar ao nascimento (média, desvio-padrão).

\begin{tabular}{lccc}
\hline & Grupo 1 & Grupo 2 & p \\
& DV normal & DV Alterado & \\
& $\mathbf{n}=\mathbf{2 6}$ & $\mathbf{n}=\mathbf{1 5}$ & \\
\hline Idade gestacional & $29 \mathrm{sem}$ & $27,6 \mathrm{sem}$ & 0,06 \\
ao nascimento & $( \pm 2,5 \mathrm{sem})$ & $( \pm 2,4 \mathrm{sem})$ & \\
Peso ao nascimento & $965 \mathrm{~g}( \pm 399 \mathrm{~g})$ & $706 \mathrm{~g}( \pm 334 \mathrm{~g})$ & $0,02^{*}$ \\
Apgar $5^{\circ}$ min $<7$ & $2(7,7 \%)$ & $3(20 \%)$ & 0,5
\end{tabular}

Dados apresentados como números absolutos por grupo ou média (e desvio-padrão). ${ }^{*} \mathrm{p}<0,05$.

$\mathrm{DV}=$ ducto venoso

As médias da IG e do peso ao nascer foram respectivamente de 29 semanas ( $\pm 2,5$ semanas) e 965 gramas ( \pm 399 gramas) nos fetos com DV normal, e de 27,6 semanas ( $\pm 2,4$ semanas) e 706 gramas ( \pm 334 gramas) naqueles com DV alterado. Foram identificados dois fetos $(7,7 \%)$ com Apgar $<7$ no grupo com DV normal e três $(21,4 \%)$ no grupo com DV alterado. Não houve diferença estatística entre os grupos quanto à IG ao nascimento $(p=0,06)$ e ao 
indice de Apgar $<7$ no $5^{\circ}$ minuto $(\mathrm{p}=0,5)$. No que diz respeito ao peso ao nascer, foi observada diferença significativa entre os dois grupos $(p=0,02)$. A Figura 1 representa a disposição gráfica da variabilidade da idade gestacional e do peso ao nascimento em relação aos grupos estudados.
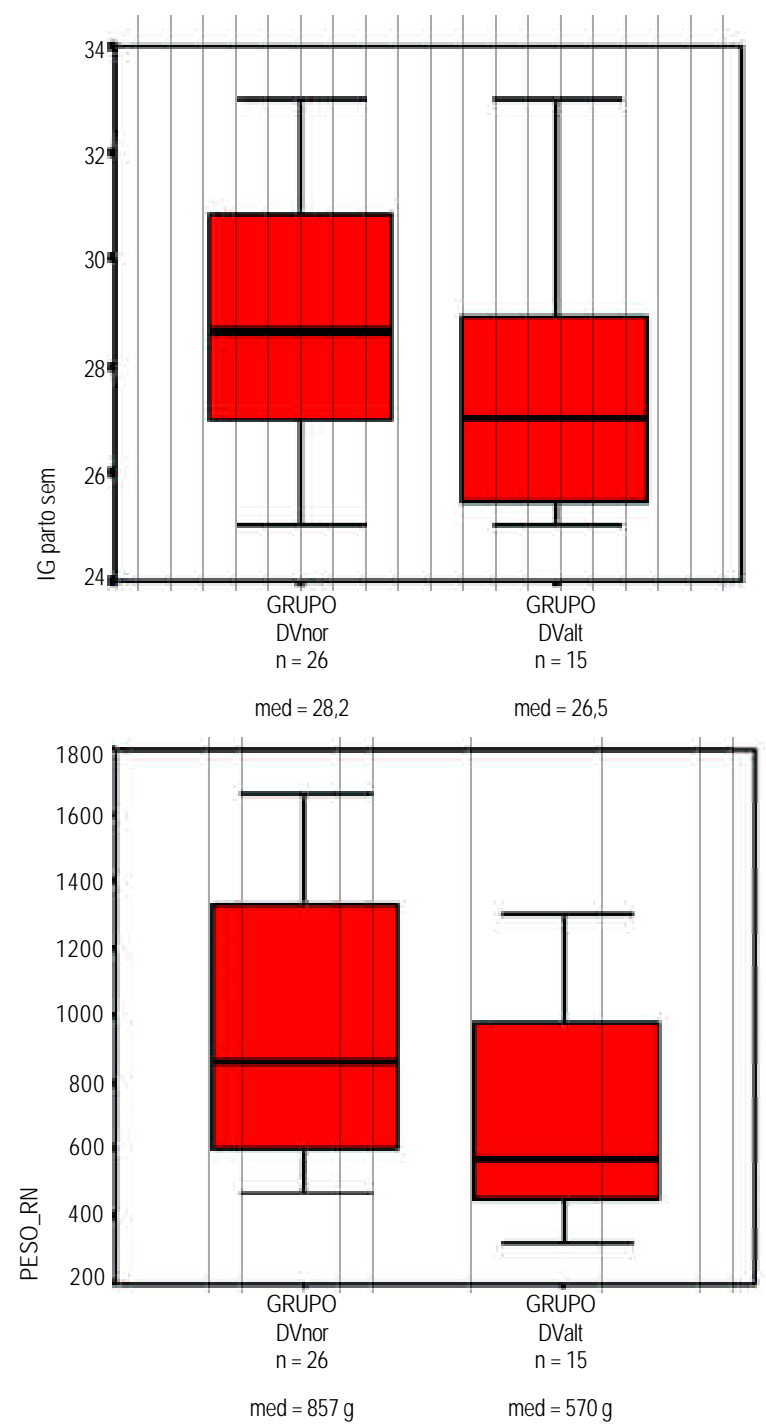

Figura 1 - Box plots da idade gestacional e do peso ao nascer em cada grupo. Representação da variabilidade (mediana e intervalo interquartil) da idade gestacional em semanas e do peso em gramas, ao nascimento, nos grupos estudados. med = mediana; DVnor = ducto venoso normal; DValt $=$ ducto venoso alterado.

Das variáveis perinatais estudadas encontrou-se uma associação estatisticamente significante entre o grupo com ducto venoso alterado e a mortalidade neonatal $(\mathrm{p}=0,049)$. Não houve casos de natimortalidade ou convulsão neonatal em ambos os grupos. Não foi identificada diferença significante entre os grupos no que diz respeito a hemorragia intraventricular $(p=0,43)$, necessidade de surfactante $(p=0,37)$, uso de respirador $(p=0,23)$, disfunção miocárdica $(p=0,22)$, enterocolite necrotizante $(p=0,43)$ e permanência na UTI por período superior ao neonatal $(\mathrm{p}=0,37)$.

Ao se excluirem da análise os três RN com peso inferior a $400 \mathrm{~g}$, não mais se observou diferença da mortalidade neonatal entre os grupos $(p=0,37)$. Não foi observada diferença significante entre os dois grupos quanto às demais variáveis estudadas, após a exclusão destes três RN. Esses dados estão representados na Tabela 2.

Tabela 2 - Resultados perinatais

\begin{tabular}{lccc}
\hline & $\begin{array}{c}\text { Grupo 1 } \\
\text { DV normal } \\
\mathbf{n = 2 6}\end{array}$ & $\begin{array}{c}\text { Grupo 2 } \\
\text { DV Alterado } \\
\mathbf{n}=\mathbf{1 5}\end{array}$ & $\mathbf{p}$ \\
\hline Morte intra-uterina & 0 & 0 & \\
Morte neonatal & 2 & 5 & $0,049^{*}$ \\
Convulsão & 0 & 0 & \\
Hemorragia intracraniana & 2 & 2 & 0,43 \\
Surfactante & 15 & 10 & 0,37 \\
Respirador & 14 & 10 & 0,23 \\
Disfunção miocárdica & 5 & 5 & 0,22 \\
Enterocolite necrotizante & 2 & 2 & 0,43 \\
Internação na UTI aos 28 & 5 & 1 & 0,37 \\
dias de vida** & & &
\end{tabular}

Dados apresentados como números absolutos por grupo.

${ }^{*} p<0,05$. **Foram excluídos desta análise os casos de mortalidade neonatal. $\mathrm{DV}=$ ducto venoso.

\section{Discussão}

Os estudos iniciais com a dopplervelocimetria do DV ocorreram no início da década de 1990, já sugerindo que o aumento da sua pulsatilidade indicaria sinais de comprometimento fetal ${ }^{14}$. Desde então, o DV tem ganhado relevância na predição de resultados perinatais adversos e na avaliação do bem-estar de fetos prematuros com CIUR e alteração de fluxo à dopplerfluxometria arterial ${ }^{19-21}$.

A alteração da relação S/A do DV tem sido apontada como sinal precoce de acidemia, que pode ocorrer até 16 dias antes de a cardiotocografia (CTG) tornar-se não reativa. A alteração da relação S/A do DV é verificada antes da ocorrência do fluxo reverso da AU, da alteração do pico da artéria pulmonar, do fluxo reverso no DV e finalmente do decréscimo da velocidade de pico da artéria aorta ${ }^{22,23}$.

A avaliação do DV em gestações de fetos com diástole zero ou reversa é considerada parâmetro adicional para determinação da interrupção da gestação e predição de resultados neonatais precoces, estando significantemente associado a alterações do $\mathrm{pH}$ arterial, excesso de base (EB) arterial, hemorragia intraventricular e mortalidade nesse período $^{24}$. Estudo recente, ao avaliar 68 gestações com CIUR, identificou que a alteração do índice de 
pulsatilidade do DV é, também, fator indicativo para internação em UTI neonatal, $\mathrm{pH}$ arterial menor que 7,10 ao nascimento e morbidade neonatal ${ }^{25}$.

No presente estudo, houve homogeneidade entre os dois grupos para IG ao nascimento e Apgar $<7$ no $5^{\circ}$ minuto. No entanto, identificou-se diferença estatisticamente significante entre os grupos no que diz respeito ao peso de nascimento $(p=0,02)$. Esse fato sugere que o déficit de peso encontrado no grupo de fetos com DV alterado pode ser resultante do maior agravo observado na distribuição do fluxo sangüíneo nestes fetos.

Observamos que o grupo de fetos com relação S/A anormal teve maior associação com o aumento da mortalidade neonatal, contudo, apresentando valor próximo ao limite $(\mathrm{p}=0,049)$. Ao retirarmos da análise os RN com peso inferior a 400 g esta associação não foi mais encontrada $(p=0,37)$. Essa observação indica que a mortalidade no grupo com DV alterado pode estar relacionada ao extremo baixo peso desses RN, no limiar da viabilidade. Não foi observada associação significativa entre a relação S/A do DV alterado com as demais variáveis estudadas.

Este estudo se diferencia de outros na literatura por associar exclusivamente a alteração do DV com variáveis perinatais desfavoráveis. Essa análise ressalta diretamente a importância da relação S/A do DV com os resultados estudados, uma vez que a interrupção da gestação ocorreu até 24 horas após a avaliação do DV. Ao contrário, outros estudos longitudinais promoveram o acompanhamento da gravidez até a alteração de outros testes biofísicos, como é o caso do perfil biofísico fetal e da cardiotocografia, sem que houvesse a insonação do DV na proximidade do parto ${ }^{7,8}$.

A abreviação do seguimento biofísico da gestação é uma possivel causa, a nosso ver, da ausência de natimortos encontrados no estudo atual e da significância estatística encontrada com a neomortalidade. A extração de fetos mais precoces resulta no nascimento de fetos vivos, porém mais prematuros, ao contrário do que encontraram Baschat et al. ${ }^{26}$ em estudo com 121 fetos com alteração de fluxo ao doppler da AU. Esses autores identificaram forte associação do DV alterado com natimortalidade, sem aumento da taxa de neomortalidade. Diferente do trabalho citado e de outros, a seleção de nossa população foi baseada em critérios rígidos de IG, envolvendo apenas fetos prematuros centralizados. Não houve inclusão de gestações a termo e daquelas acometidas apenas com alteração de fluxo na $\mathrm{AU}^{26-28}$. A seleção da população teve por objetivo restringir o uso do método a situações de real importância na prática obstétrica.

Estudo multicêntrico com 70 fetos portadores de CIUR precoce com IG variando entre 26 e
33 semanas de gestação encontrou correlação entre índices alterados do DV e resultados perinatais adversos, como mortalidade perinatal, hemorragia intracraniana e broncodisplasia pulmonar ${ }^{21}$. Nossos resultados se aproximam destes autores somente no que tange à mortalidade neonatal. Fato semelhante é verificado quando confrontamos nosso trabalho com outro nacional, onde foi identificada a associação do índice de pulsatilidade para veias do DV, com índices de Apgar no $1^{\circ}$ e $5^{\circ}$ minuto inferioriores a sete, acidemia ao nascimento, necessidade de UTI neonatal e óbito neonatal em 108 gestações de alto risco ${ }^{29}$. Outro estudo prospectivo avaliou 42 fetos prematuros com CIUR, que foram divididos em dois grupos a partir do resultado da relação (S/A)/S. Não foi encontrada associação entre o DV alterado e resultados neonatais, tais como: morte fetal, enterocolite necrotizante, hemorragia ventricular e síndrome da membrana hialina, em fetos com menos de 32 semanas. Foi observada, no entanto, diferença significativa entre os grupos, no que diz respeito ao peso ao nascer, à semelhança do encontrado em nossos resultados. A principal limitação dos autores foi o pequeno tamanho da amostra ${ }^{30}$.

Os nossos resultados sugerem que a anormalidade do fluxo no DV detectado à dopplervelocimetria não está associada a resultados perinatais adversos outros, além da mortalidade neonatal. A associação destas duas variáveis pode ser considerada estatisticamente significante, porém com valor próximo ao limite $(\mathrm{p}=0,049)$. Em nossa análise, esse dado ganha ainda mais consistência se considerarmos apenas os fetos com peso ao nascer superior a $400 \mathrm{~g}$. Desta forma, a alteração da relação S/A do DV não apresentou, em nosso trabalho, associação com mortalidade perinatal em fetos prematuros viáveis. O tamanho inadequado da população inviabiliza outras associações com a alteração da relação S/A do ducto venoso.

\section{Referências}

1. Romero R, Kalache KD, Kadar N. Timing the delivery of the preterm severely growth-restricted fetus: venous Doppler, cardiotocography or the biophysical profile? Ultrasound Obstet Gynecol. 2002;19(2):118 -21.

2. Schwarze A, Gembruch U, Krapp M, Katalinic A, Germer U, Axt-Fliedner R. Qualitative venous Doppler flow waveform analysis in preterm intrauterine growthrestricted fetuses with ARED flow in the umbilical artery - correlation with short-term outcome. Ultrasound Obstet Gynecol. 2005;25(6):573-9.

3. Neilson JP, Alfirevic Z. Doppler ultrasound for fetal assessment in high risk pregnancies. Cochrane Database Syst Rev. 2002;(3):CD000073. 
4. Westergaard HB, Langhoff-Roos J, Lingman G, Marsal K, Kreiner S. A critical appraisal of the use of umbilical artery Doppler ultrasound in high-risk pregnancies: use of meta-analyses in evidence-based obstetrics. Ultrasound Obstet Gynecol. 2001;17(6): 466-76.

5. Wladimiroff JW, Van Der Wijngaard JA, Degani S, Noordam MJ, van Eyck J, Tonge HM. Cerebral and umbilical arterial blood flow velocity waveforms in normal and growth-retarded pregnancies. Obstet Gynecol. 1987;69(5):705-9.

6- Baschat AA. Doppler application in the delivery timing of the preterm growth- restricted fetus: another step in the right direction. Ultrasound Obstet Gynecol. 2004;23(2):111-8.

7. Ferrazzi E, Bellotti M, Bozzo M, Rigano S, Pardi G, Battaglia $F$, et al. The temporal sequence of changes in fetal velocimetry indices for growth-restricted fetus. Am J Obstet Gynecol. 2000;182(1 Pt 2):S17.

8. Baschat AA, Gembruch U, Harman CR. The sequence of changes in Doppler and biophysical parameters as severe fetal growth restriction worsens. Ultrasound Obstet Gynecol. 2001;18(6):571-7.

9. Sá RAM, Netto HC, Lopes LM, Barreto MJV, Cabral ACV. Dopplerfluxometria do ducto venoso - relação com a gasometria em fetos prematuros com centralização de fluxo sangüineo. Rev Bras Ginecol Obstet. 2003;25(4):261-8.

10. Carvalho FHC, Moron AF, Mattar R, Murta CGV, Santana RM, Torloni MR, et al. Índices veno-arteriais para predição da acidemia fetal ao nascimento em gestações com insuficiência placentária. Rev Bras Ginecol Obstet. 2004;26(8):641-7.

11. Rizzo G, Capponi A, Talone PE, Arduini D, Romanini C. Doppler indices from inferior vena cava and ductus venosus in predicting $\mathrm{pH}$ and oxygen tension in umbilical blood at cordocentesis in growth-retarded fetuses. Ultrasound Obstet Gynecol. 1996;7(6):401-10.

12.Arduini D, Rizzo G. Normal values of pulsatility index from fetal vessels: a cross-sectional study on 1556 healthy fetuses. J Perinat Med. 1990;18(3):165-72.

13. Mari G, Deter RL. Middle cerebral artery flow velocity waveforms in normal and small-for-gestational-age fetuses. Am J Obstet Gynecol. 1992;166(4):1262-70.

14. Kiserud T, Eik-Nes SH, Blaas HG, Hellevik LR. Ultrasonographic velocimetry of the fetal ductus venosus. Lancet. 1991;338 (8780):1412-4.

15. Hecher K, Campbell S, Doyle P, Harrington K, Nicolaides K. Assessment of fetal compromise by Doppler ultrasound investigation of fetal circulation: arterial, intracardiac, and venous blood flow velocity studies. Circulation. 1995;91(1):129-38.

16. Rizzo G, Arduini D, Romanini C. Doppler echocardiographic assessment of fetal cardiac function. Ultrasound Obstet Gynecol. 1992;2(6):434-45.

17. Baschat AA. Relationship between placental blood flow resistance and precordial venous Doppler indices. Ultrasound Obstet Gynecol. 2003;22(6):561-6.

18. Sá RAM, Netto HC, Amim J Jr, Silva NR, Bornia RBRG, Franco MJ, et al. Ductus venosus velocimetry in normal pregnancy. Int J Gynaecol Obstet. 2000;70 Suppl 1:A28.

19. Ozcan T, Sbracia M, D’Ancona RL, Copel JA, Mari G. Arterial and venous Doppler velocimetry in the severely growth-restricted fetus and associations with adverse perinatal outcome. Ultrasound Obstet Gynecol. 1998;12(1):39-44.

20. Hecher K, Bilardo CM, Stigter RH, Ville Y, Hackeloer BJ, Kok HJ, et al. Monitoring of fetuses with intrauterine growth restriction: a longitudinal study. Ultrasound Obstet Gynecol. 2001;18(6):564-70.

21. Bilardo CM, Wolf H, Stigter RH, Ville Y, Baez E, Visser GH, et al. Relationship between monitoring parameters and perinatal outcome in severe, early intrauterine growth restriction. Ultrasound Obstet Gynecol. 2004;23(2):119-25.

22.Ferrazzi E, Bozzo M, Rigano S, Bellotti M, Morabito A, Pardi G, et al. Temporal sequence of abnormal Doppler changes in the peripheral and central circulatory systems of the growth-restricted fetus. Ultrasound Obstet Gynecol. 2002;19(2):140-6.

23. Hecher K, Hackeloer BJ. Cardiotocogram compared to Doppler investigation of the fetal circulation in the premature growth-retarded fetus: longitudinal observations. Ultrasound Obstet Gynecol. 1997;9(3):152-61.

24. Müller T, Nanan R, Rehn M, Kristen P, Dietl J. Arterial and ductus venosus Doppler in fetuses with absent or reverse end-diastolic flow in the umbilical artery: correlation with short-term perinatal outcome. Acta Obstet Gynecol Scand. 2002;81(9):860-6.

25. Figueras F, Martínez JM, Puerto B, Coll O, Cararach $\mathrm{V}$, Vanrell JA. Contraction stress test versus ductus venosus Doppler evaluation for the prediction of adverse perinatal outcome in growth-restricted fetuses with non-reassuring non-stress test. Ultrasound Obstet Gynecol. 2003;21(3):250-5.

26. Baschat AA, Gembruch U, Reiss I, Gortner L, Weiner CP, Harman CR. Relationship between arterial and venous Doppler and perinatal outcome in fetal growth restriction. Ultrasound Obstet Gynecol. 2000;16(5):407-13.

27. Müller T, Nanan R, Rehn M, Kristen P, Dietl J. Arterial and ductus venosus Doppler in fetuses with absent or reverse end-diastolic flow in the umbilical artery: longitudinal analysis. Fetal Diagn Ther. 2003;18(3):163-9.

28. Baschat AA, Güclü S, Kush ML, Gembruch U, Weiner C, Harman CR. Venous Doppler in prediction of acid-base status of growth-restricted fetuses with elevated placental blood flow resistance. Am J Obstet Gynecol. 2004;191(1):277-84.

29. Andrade JQ, Miyadahira S, Namura RMY, Francisco RPV, Zugaib M. Dopplervelocimetria dos compartimentos arterial e venoso da circulação fetal e umbilical em gestação de alto-risco: análise dos resultados perinatais. Rev Bras Ginecol Obstet. 2002;24(3):153-60.

30.Ritter S, Jörn H, Weiss C, Rath W. Importance of ductus venosus Doppler assessment for fetal outcome in cases of intrauterine growth restriction. Fetal Diagn Ther. 2004;19(4):348-55. 